\title{
Error Analysis of Quartz Crystal Resonator Applications
}

\author{
Ralf Lucklum ${ }^{1)}$, C. Behling ${ }^{1)}$, P. Hauptmann ${ }^{1)}$, R.W. Cernosek ${ }^{2}$, and S.J. Martin ${ }^{2)}$ \\ M⿻1 03193 \\ ${ }^{1)}$ Otto-von-Guericke-University, IPE, P.O.B. 4120, D-39016 Magdeburg, Germany \\ 2) Sandia National Laboratories, MR\&DD, P.O.B. 5800, Albuquerque, NM 87185-1425, USA
}

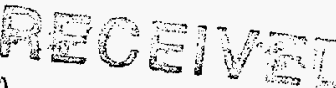

\section{SUMMARY}

Quartz crystal resonators in chemical sensing applications are usually configured as the frequency determining element of an electrical oscillator. By contrast, the shear modulus determination of a polymer coating needs a complete impedance analysis. The first part of this contribution reports the error made if common approximations are used to relate the frequency shift to the sorbed mass. In the second part we discuss different error sources in the procedure to determine shear parameters.

Keywords: Acoustic Sensors, Chemical Sensors, Material Parameter Determination

\section{INTRODUCTION}

Acoustic wave devices are being used increasingly as chemical sensors and for the determination of polymer shear parameters. The simplest and most common application is the gravimetric quartz crystal microbalance [1]. Here, a change in the surface mass is proportional to the shift in the resonant frequency of a quartz crystal resonator. This simple relation is restricted to thin rigid layers. For chemical applications, a sensitive and selective material must be coated on one or both surfaces of the quartz that interacts with the analyte of interest. Many of these layers do not fulfill the requirements of the pure gravimetric principle. Moreover, analyte sorption may cause both a mass increase and a change in the material parameters of the coating. Under these conditions different mechanisms determine the sensor response. In the first part of this paper we focus on the errors resulting from common approximations under different experimental conditions.

Under certain circumstances material parameters of the coating contribute significantly to the sensor response. This effect can be exploited for the determination of the shear parameters of the coating. This application requires impedance analysis and a complex fitting procedure. A new principle based on a dual-line resonator which overcomes these disadvantages will be published soon. In the second part of this paper we discuss the error influences of the computational procedures to determine material parameters.

\section{THEORY}

\section{Acoustics}

The vibration behavior of a quartz crystal and the wave propagation in the system quartz-coatingmedium can be derived from a one-dimensional solution of the wave equation. To calculate the overall system characteristics, the chain matrix technique of the transmission line model with two acoustic ports and one electrical port is used. A more detailed discussion is presented in [2]. Important results can be summarized as follows:

- The acoustic load (surface mechanical impedance) of a coating with finite thickness is a function of the coating density, $\rho_{c}$, coating thickness, $d_{c}$, angular frequency, $\omega$, and the coating shear modulus, $\mathrm{G}_{\mathrm{c}}$ :

$$
Z_{s}=j \sqrt{\rho_{c} G_{c}} \tan \left(\omega \sqrt{\frac{\rho_{c}}{G_{c}}} d_{c}\right)
$$

- Viscoelasticity can be expressed by a complex shear modulus:

$$
\mathrm{G}_{\mathrm{c}}=\mathrm{G}_{\mathrm{c}}^{\prime}+\mathrm{jG}_{\mathrm{c}}^{\prime \prime}
$$

- The impedance measured at the electrical port is a parallel combination of the static crystal capacitance and the system motional impedance.

- The motional impedance can be derived from $Z_{\mathrm{s}}$ and the quartz parameters and separated into two parts, the impedance of the uncoated quartz and the impedance generated by the acoustic load.

- Near resonance the impedance of the uncoated quartz can be transformed into the Butterworth 


\section{DISCLAIMER}

This report was prepared as an account of work sponsored by an agency of the United States Government. Neither the United States Government nor any agency thereof, nor any of their employees, makes any warranty, express or implied, or assumes any legal liability or responsibility for the accuracy, completeness, or usefulness of any information, apparatus, product, or process disclosed, or represents that its use would not infringe privately owned rights. Reference herein to any specific commercial product, process, or service by trade name, trademark, manufacturer, or otherwise does not necessarily constitute or imply its endorsement, recommendation, or favoring by the United States Government or any agency thereof. The views and opinions of authors expressed herein do not necessarily state or reflect those of the United States Government or any agency thereof. 


\section{DISClaAmER}

Portions of this document may be illegible in electronic image products. Images are produced from the best available original document. 
Van-Dyke (BVD) equivalent circuit.

- For small loads the electrical load impedance is proportional to the acoustic load (small load approximation).

- For rigid and sufficiently thin layers, the acoustic impedance is proportional to the surface mass, $\rho_{c} d_{c}$, and independent of shear parameters, hence the frequency shift is proportional to the surface mass (Sauerbrey approximation).

- In case of low energy dissipation the frequency shift is proportional to the imaginary part of $Z_{s}$ (impedance approximation).

\section{Material Parameters}

Most coating materials used for chemical sensing exhibit viscoelastic behavior. The dynamic properties are characterized by a relaxation time, $\tau$. When $\tau$ is much longer than the period of the crystal oscillation, the relaxation is 'frozen'. The coating behaves like a glass. If a viscoelastic material is deformed on a time scale larger than the relaxation time, the coating material behaves like a rubber.

The glass transition temperature is frequency dependent, increasing with frequency (WLF formalism [3]).

Analyte sorption may cause swelling and plasticization and reduces the glass transition temperature [4].

\section{RESULTS AND DISCUSSION}

The following results are based on calculations with the full one-dimensional transmission line model, the impedance approximation as well as Sauerbrey's equation for a quartz resonator with a single coating. For the polymer shear parameter determination, a lumped-element equivalent circuit model was also used. The following notation and values are applied in the text and the figures of this section:

Tab. 1 List of abbreviations

\begin{tabular}{|cc|}
\hline$h$ - glassy & $1-$ thin \\
$\quad G^{\prime}=10^{9} \mathrm{~Pa}, G^{\prime \prime}=10^{8} \mathrm{~Pa}$ & $\mathrm{~d}_{\mathrm{c}}=200 \mathrm{~nm}$ \\
$\mathrm{~s}-$ & $2-$ medium \\
rubbery & $\mathrm{d}_{\mathrm{c}}=500 \mathrm{~nm}$ \\
$\mathrm{G}^{\prime}=10^{6} \mathrm{~Pa}, \mathrm{G}^{\prime \prime}=10^{6} \mathrm{~Pa}$ & $3-$ thick \\
$\mathrm{fm}$-resonance frequency & $\mathrm{d}_{\mathrm{c}}=800 \mathrm{~nm}$ \\
at impedance minimum & fs - series zero phase \\
$\mathrm{fn} \quad$ resonance frequency & resonance \\
at impedance maximum & fp parallel zero phase \\
$\mathrm{f} \mathrm{Im}(\mathrm{Z})$ & resonance \\
impedance approximation &
\end{tabular}

\section{Coating procedure}

Figure 1 summarizes the resonator frequency shifts due to coating the quartz on one surface. The different models yield minor differences for coatings with a rubbery material of medium thickness, s2, and major differences for a thick rubbery layer, s3. In all other cases the approximation error is less than $2.5 \%$, equivalent to a thickness error less than $5 \mathrm{~nm}$ for the thin coating, $12.5 \mathrm{~nm}$ for the medium coating and $20 \mathrm{~nm}$ for a thick coating.

In the case of a $500 \mathrm{~nm}$ rubbery coating, s2, the frequency shifts differ from each other. The frequency shift at zero phase is smaller than the frequency shift at impedance minimum. Sauerbrey's equation predicts a frequency shift which is already $14 \%$ too small. The impedance approximation fits better with a value between $\mathrm{fm}(\mathrm{fn})$ and $\mathrm{fs}(\mathrm{fp})$.

For a thick and rubbery coating, s3, phase shift and attenuation of the propagating wave contribute significantly to the measured frequency shift. It also depends heavily on the point where the electrical oscillator works. This is mainly caused by a flattening of the impedance curve. From Sauerbrey we would expect a frequency shift smaller than the frequency shift at impedance minimum. In other words, the coating would be too thin (20\%) if the oscillator works at that point and Sauerbrey's equation is applied.

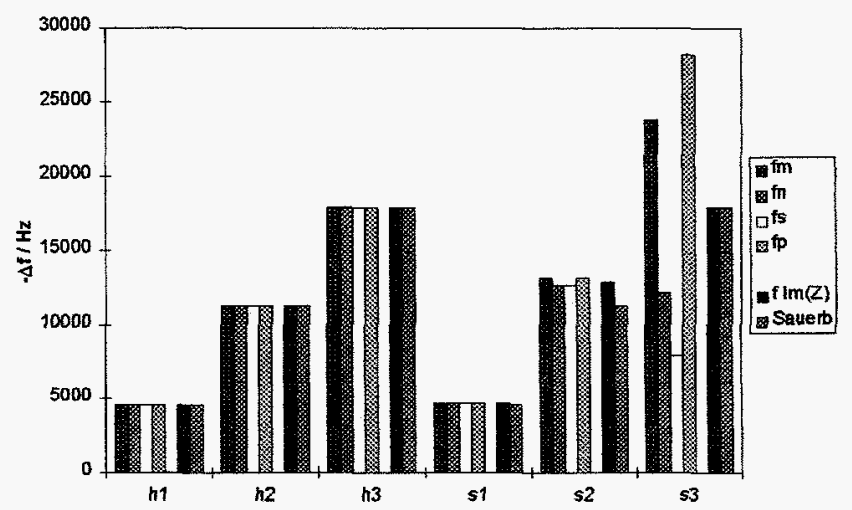

Fig. 1: Frequency shift after coating a $10 \mathrm{MHz}$ resonator on one side (for abbr. see text)

Thus, Sauerbrey's equation should be sufficient for an estimation of the coating thickness of a glassy coating or a thin rubbery coating, but the impedance approximation is necessary in case of a medium rubbery coating. If the coating is thick and of rubberlike consistency, the operating point of the oscillator must be known for a correct interpretation of the frequency shift. 


\section{Chemical application}

As a first step, we assume that the analyte sorption does not significantly influences the shear parameters, $G_{c}$ of the coating. A relatively small deviation of $10 \%$ in $G_{c}$ results in a frequency shift not more than a few $\mathrm{Hz}$. Only for a thick rubbery coating does the contribution from shear parameters shift the frequency in excess of $1 \mathrm{kHz}$.

Fig. 2 summarizes the decrease in frequency after analyte exposure compared to the respective frequencies after coating. The glassy polymer coating and the thin rubbery polymer coating do not create dramatic differences in the model predictions. The experimenter must decide whether model deviations, up to $8 \%$ in the calculated mass increase, are negligible. Although pure mass accumulation is the major contributor to the frequency change, the slope of Sauerbrey's equation is smaller than the real relation between mass increase and frequency decrease for a rubbery material. As one can see in Figure 2 for s2, the sensitivity to mass accumulation increases in a given thickness range for a rubbery coating material.

The thick rubbery coating shows unexpected positive frequency shifts. This results from both a phase shift of the acoustic wave in the coating and a significant damping. The sensor response depends heavily on the operating point of the oscillator. This configuration is not well adapted to analytical applications.

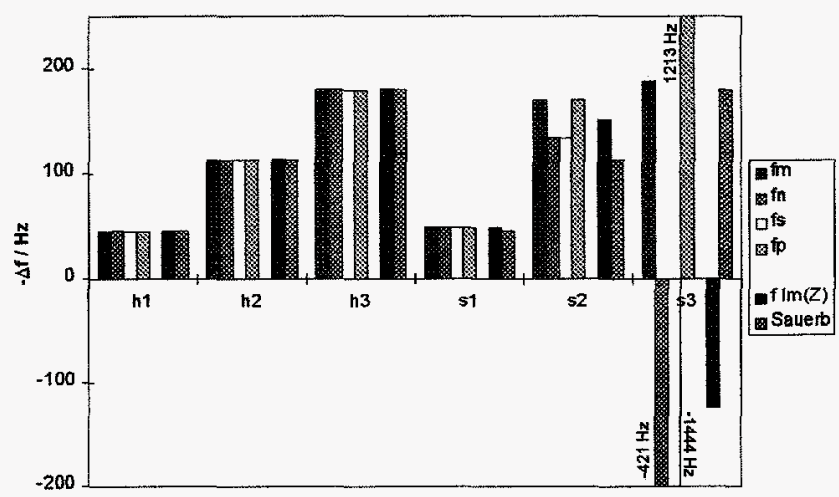

Fig. 2: Frequency shift after analyte sorption with only $1 \%$ mass accumulation

In the second step we consider major changes in the shear modulus of the coating. If analyte sorption causes a phase transition from a glasslike to a rubberlike consistency, the shear modulus changes as much as a few orders of magnitude. The consequence for the measured frequency shift is shown in Figure 3 for the thin coating. The modulus effect exceeds the pure mass effect (Sauerbrey column) increasingly as the material gets rubbery. The impedance approximation allows a rough estimate as long as the shear parameters and the working point of the oscillator are known. Although we calculated huge frequency changes, a controlled reproducible use of this 'material amplification' of the mass effect seems to be problematic in practice.

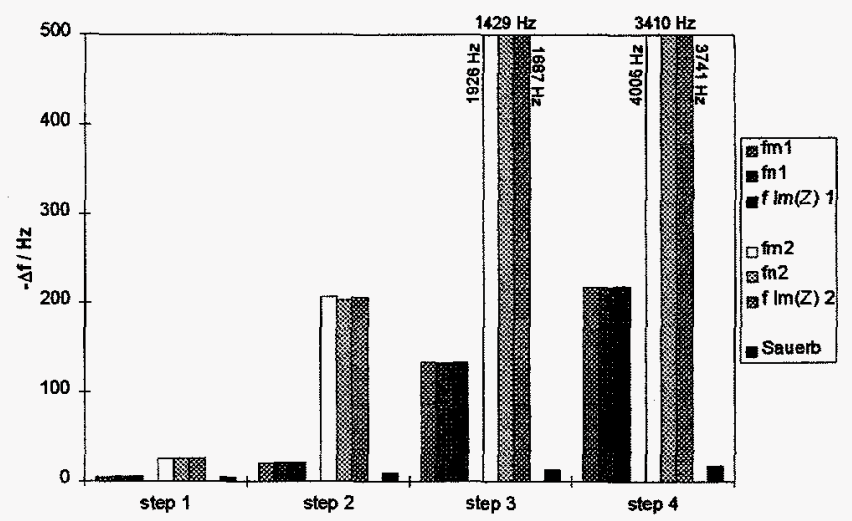

Fig. 3: Frequency shift after analyte sorption with both mass accumulation ( $0.1 \%$ per step) and phase transition

\section{Shear parameter determination}

In an initial step the effective quartz parameters were determined from the impedance curve of an uncoated resonator. The coating thickness was calculated from the frequency shift at $-50^{\circ} \mathrm{C}$. Figure 4 illustrates the extracted shear storage modulus, $\mathrm{G}^{\prime}(=\mathrm{G} 1)$, and shear loss modulus, $\mathrm{G}^{\prime \prime}(=\mathrm{G} 2)$, of polyisobutylene (PIB). The curves show the phase transition from the glassy to a rubbery state. The dynamic glass transition temperature is much higher than the static glass transition temperature $\left(-68^{\circ} \mathrm{C}\right)$, i. e., the coating material which is rubbery at $20^{\circ} \mathrm{C}$ behaves like a glassy material for the probing acoustic wave at this temperature.

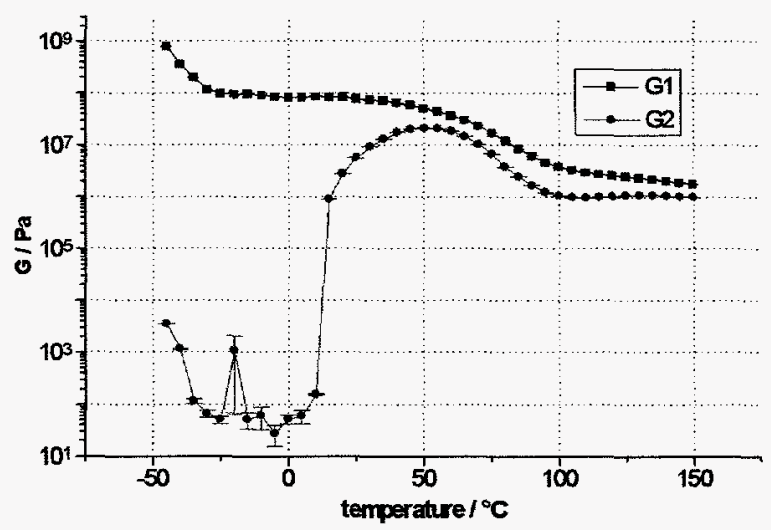

Fig. 4: Extracted shear parameters for a PIB coating The error bars indicate a fitting error of $1 \mathrm{ppm}$. It is not surprising that the principle works better for rubbery coatings. In the glassy state the small load 
assumptions applies. The argument of the tanfunction in equ. 1 becomes small (high $G^{\prime}$ ) and essentially real $\left(\mathrm{G}^{\prime \prime}<<\mathrm{G}^{\prime}\right)$, and $\tan (\mathrm{x})$ is approximated by $x$ and the $G$-dependence cancels out.

Figure 5 shows a part of the fitting error field for the $0^{\circ} \mathrm{C}$ measurement. The darker the gray the better the fit. It is obvious that the ability of implemented

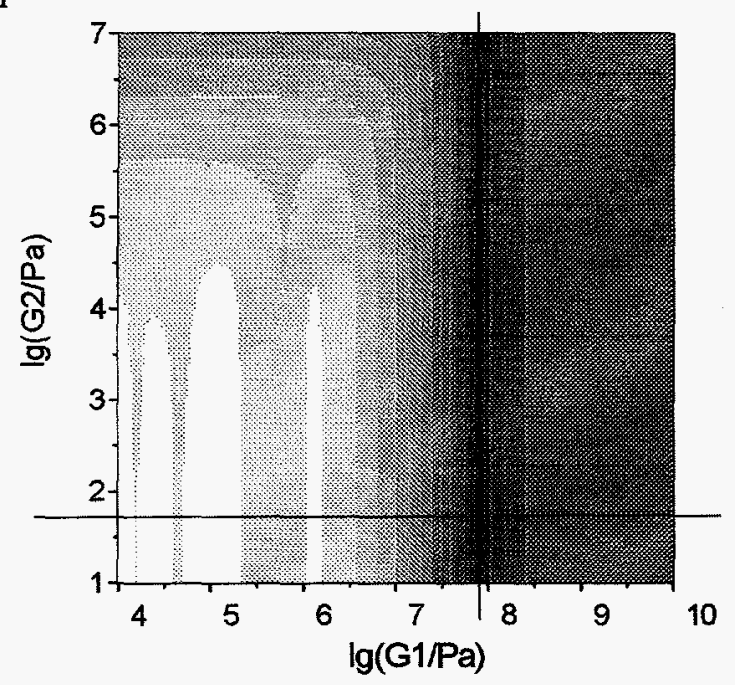

Fig. 5: Part of the fitting error field at $0^{\circ} \mathrm{C}$

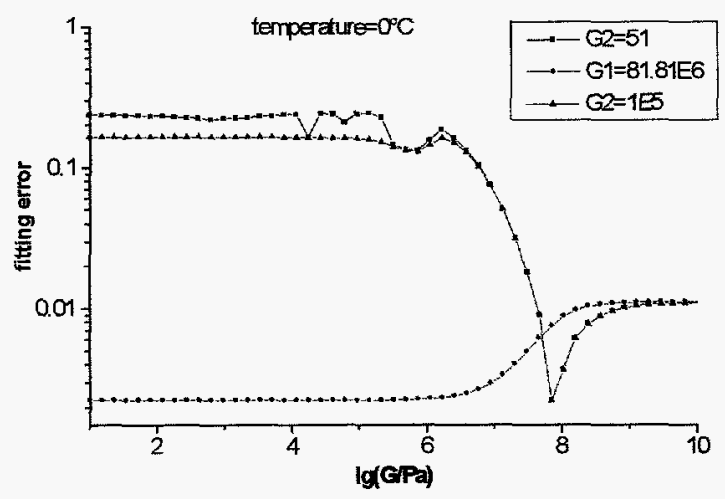

Fig. 6: Fitting error curve at the position marked in Fig. 5 algorithm to find the best fit is strongly influenced by the number of measurement points and the search routine. The thin black area at $\mathrm{G}^{\prime} \approx 10^{8} \mathrm{~Pa}$ indicates why the extracted value for $G^{\prime \prime}$ is much too small. The fitting error at the expected value of $10^{5} \mathrm{~Pa}$ is only slightly higher than that at the computed value. It is shown in Figure 6. While the fitting error for $G$ ' has a clear minimum, no 'real' minimum exists for $G^{\prime \prime}$. Moreover, the calculated value for $G^{\prime}$ does not change if $G^{\prime \prime}$ is set to $10^{5} \mathrm{~Pa}$. The situation improves for the rubbery state. The fitting procedure finds a well defined minimum for $G^{\prime}$ and $G^{\prime \prime}$.

The measured or calculated coating thickness is the most important geometric parameter of the fitting procedure and must be determined with high accuracy. Figure 7 demonstrates the result of a $0.1 \%$ and a $0.5 \%$ decrease in the coating thickness. While in the rubbery state, the extracted values for $G^{\prime}\left(G^{\prime \prime}\right)$ are almost the same, but the error increases to $35(60) \%$ in the transition area and $55(60) \%$ in the glassy state.

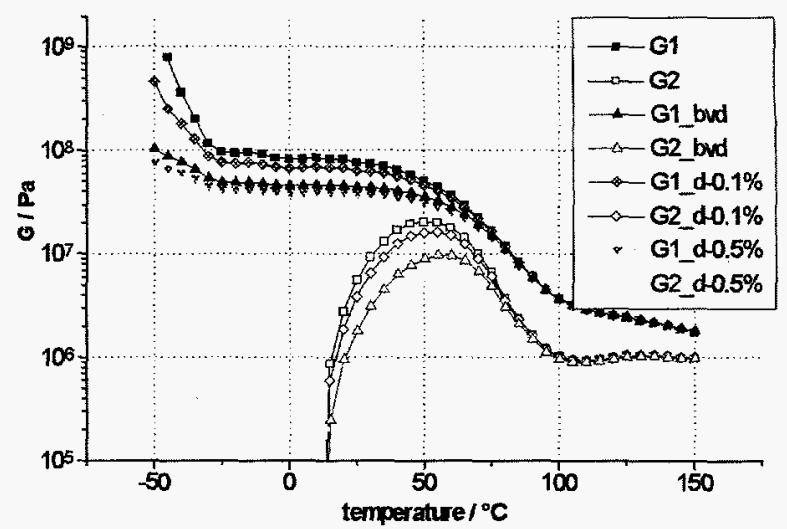

Fig. 7:Dependence of the extracted shear parameters on the coating thickness and model used

Finally, the shear parameter curves obtained from the lumped element model (BVD) with the small load approximation are also shownin Figure 7 . The values are smaller than the results extracted with the full transmission line model in the glassy area and similar in the rubbery state.

\section{Acknowledgment}

This work was supported by the Ministry for Education and Research (BMBF) under Contract 01 RA 9603/1 and German Research Foundation (DFG) under Contract Lu605/2-1. Part of the work was performed at Sandia National Laboratories and supported by the United States Department of Energy under Contract DE-AC0494AL85000. Sandia is a multiprogram laboratory operated by Sandia Corporation, a Lockheed Martin Company, for the United States Department of Energy.

\section{References}

[1] G. Sauerbrey, Z. Phys. 155, 206-22 (1955).

[2] R. Lucklum, C. Behling, R.W. Cernosek, S.J. Martin, J. Appl. Phys. 30, 346-56 (1997).

[3] M.L. Williams, R.F. Landel, J.D. Ferry, J. Am. Chem. Soc. 77, 3701-07 (1955)

[4] S.J. Martin, G.C. Frye, S.D. Senturia, Anal. Chem. 64, 2201-19 (1994). 